\title{
Meson correlation functions at high temperatures
}

\author{
S. Wissel*, E. Laermann, S. Shcheredin \\ Universität Bielefeld \\ Fakultät für Physik \\ Universitätsstrasse 25 \\ D-33615 Bielefeld, Germany \\ E-mail: \\ wisselaphysik.uni-bielefeld.de, \\ edwin@physik. uni-bielefeld.de, \\ shcheredephysik.uni-bielefeld.de
}

\section{S. Datta, F. Karsch}

Brookhaven National Lab

PO Box 5000

Phys. Dept., Bldg. 510C

Upton, NY 11973-5000

USA

E-mail:

saumen@quark • phy · bnl · gov

karsch@quark ·phy ·bnl.gov

We present preliminary results for the correlation- and spectral functions of different meson channels on the lattice. The main focus lies on gaining control over cut-off as well as on the finite-volume effects. Extrapolations of screening masses above the deconfining temperature are guided by the result of the free $(T=\infty)$ case on the lattice and in the continuum. We study the quenched non-perturbatively improved Wilson-clover fermion as well as the hypercube fermion action which might show less cut-off effects.

XXIIIrd International Symposium on Lattice Field Theory

25-30 July 2005

Trinity College, Dublin, Ireland

${ }^{*}$ Speaker. 


\section{Introduction}

An important goal of contemporary physics is to understand the nature of excitations characterizing the structure of QCD at high temperatures. At large temperatures, the running coupling constant $g(T)$ becomes small and therefore one can expect to see a weakly interacting gas of quarks and gluons. However, at temperatures up to a few times $T_{c}$ some bound states might survive the deconfinement transition.

Due to the small temporal extension of the lattice, it is a difficult task to reliably extract information on bound states from temporal correlators. Like most investigations so far, we first have studied spatial correlation functions, but have attempted to carry out the infinite volume as well as the continuum limit. Second, we employed the Maximum Entropy Method (MEM) to extract information from the temporal correlators, working with and comparing two different fermion discretizations with different UV cut-off effects.

\section{Simulation details}

The results presented here are based on quenched gauge field configurations generated with the standard Wilson plaquette gauge action. The physical (temperature) scale has been obtained using the interpolation formula of [1] for the string tension. In order to study finite volume and lattice spacing effects, at all considered temperatures, we have generated gauge field configurations at three different volumes and lattice spacings.

\begin{tabular}{|c|c|c|c|c|l|c|}
\hline$T / T_{c}$ & $\beta$ & $N_{\sigma}^{3} \times N_{\tau}$ & $a[\mathrm{fm}]$ & $N_{\text {conf }}$ & $\kappa$ & $\kappa_{c}$ \\
\hline 1.24 & 6.205 & $(64|32| 24 \mid 16)^{3} \times 8$ & 0.075 & $(46|61| 85 \mid 89)$ & 0.13599 & 0.13580 \\
1.24 & 6.499 & $(48|36| 24)^{3} \times 12$ & 0.049 & $(81|81| 78)$ & 0.13558 & 0.13558 \\
1.24 & 6.721 & $(64|48| 32)^{3} \times 16$ & 0.038 & $(46|96| 81)$ & 0.13507 & 0.13522 \\
1.19 & 6.872 & $64^{3} \times 20$ & 0.031 & 120 & 0.13494 & 0.13499 \\
\hline 1.49 & 6.338 & $(64|32| 24 \mid 16)^{3} \times 8$ & 0.062 & $(46|62| 59 \mid 60)$ & 0.13581 & 0.13578 \\
1.49 & 6.640 & $(48|36| 24)^{3} \times 12$ & 0.041 & $(61|59| 68)$ & 0.13536 & 0.13535 \\
1.49 & 6.872 & $(64|48| 32)^{3} \times 16$ & 0.031 & $(66|62| 60)$ & 0.13495 & 0.13499 \\
1.46 & 7.192 & $64^{3} \times 24$ & 0.021 & 80 & 0.13440 & 0.13450 \\
\hline 2.98 & 6.872 & $(96|64| 32|24| 16)^{3} \times 8$ & 0.031 & $(37|43| 65|80| 80)$ & 0.13494 & 0.13499 \\
2.91 & 7.192 & $(48|36| 24)^{3} \times 12$ & 0.021 & $(85|80| 80)$ & 0.13440 & 0.13450 \\
2.98 & 7.457 & $(64|48| 32)^{3} \times 16$ & 0.015 & $(80|80| 60)$ & 0.13390 & 0.13394 \\
\hline 5.96 & 7.457 & $32^{3} \times 8$ & 0.015 & 70 & 0.13390 & 0.13394 \\
\hline
\end{tabular}

Table 1: Simulation parameters for the underlying study. (Data of the hypercube action are marked in red)

We use two different types of fermions: non-perturbatively $\mathscr{O}(a)$ improved [2] Wilson-clover fermions [3] and hypercube fermions [4]. At temperatures sufficiently above $T_{c}$ one is able to perform the Wilson matrix inversions in the chiral limit since the zero modes are absent or very suppressed. We therefore choose to work at or close to $\kappa_{c}$ values, determined and interpolated at $T=0$ from [2], see Tab. 1 .

An alternative approach is to use the fixed point perfect action, which is free from any cut-off effects. The existence of such an action is a consequence of the Wilsons renormalization group 
theory [5]. For free quarks the perfect action is known analytically and can be obtained with a technique called "blocking from the continuum" $[6,7]$. However on a lattice one truncates the perfect action to a unit hypercube to render it ultralocal. To gauge the action we employed a simple ansatz for the interacting gauge fields called the hyperlinks. We supplemented this construction by fat links and an additional enhancement of the operator couplings for the free perfect action. These provide the hypercube operator with better chiral properties which are controlled by the shape of the eigenvalue spectrum [4]. The latter is adjusted to hit the chiral limit for the quark masses.

\section{Screening masses above $T_{c}$}

In the infinite temperature limit mesonic correlation functions are combinations of two free quark propagators. Correspondingly at large distances the decay of spatial correlators is dominated by twice the lowest quark Matsubara frequency,

$$
m_{s c r}^{\text {meson }}=2 \omega_{0}^{\text {quark }}=2 \pi T .
$$

In the continuum the spatial correlation function of the free quark-antiquark pair with pion quantum numbers is [8]

$$
G_{\pi}(z)=\frac{N_{c} T}{2 \pi z^{2} \sinh (2 \pi T z)}[1+2 \pi T z \operatorname{coth}(2 \pi T z)] .
$$

From this an effective z-dependent screening mass can be defined by

$$
m(z)=-\frac{1}{G(z)} \frac{\partial G(z)}{\partial z}=2 \pi T\left\{\frac{1}{x}(2+x \operatorname{coth}(x))+\frac{1}{1+x \operatorname{coth}(x)}\left(\frac{x}{\sinh ^{2}(x)}-\operatorname{coth}(x)\right)\right\}
$$

where $x=2 \pi T z$. Note that the effective screening mass, which is shown in Fig. 1 (left), does not exhibit any plateau-like behavior, which otherwise would signal the presence of a genuine pole contribution. The anticipated value $2 \pi T$ is reached from above, yet only at asymptotically large distances. Also in the interacting case a genuine plateau can not be identified at temperatures above $T_{c}$ for our lattices. For definiteness we therefore will quote screening masses obtained at a certain spatial separation. The choice $z=L / 4$ then leads in the free case to

$$
\frac{m(z=L / 4)}{T}=2 \pi\left(1+\frac{2}{\pi} \frac{1}{L T}+\ldots\right)
$$

i.e. the leading correction to the continuum screening mass is linearly dependent, $\sim 1 /(L T)$, on the (inverse) separation.

In a numerical lattice investigation the separation is limited by the box size. In order to address finite volume effects we have calculated the free spatial lattice correlation functions semianalytically [9]. It turns out, Fig. 1, that also in this case the leading finite volume correction to the screening mass is linearly dependent on $1 /(L T)=N_{\tau} / N_{\sigma}$. In the interacting case the finite volume effects are less severe. This can be understood because freely propagating quarks feel the box boundaries (and the momentum cut-off) more strongly than interacting quarks. In order to describe the volume effects we have therefore taken the exponent of the $1 /(L T)$ term as a free parameter,

$$
\frac{m(L, a)}{T}=\frac{m(a)}{T}\left(1+\gamma\left(\frac{N_{\tau}}{N_{\sigma}}\right)^{p}\right)
$$



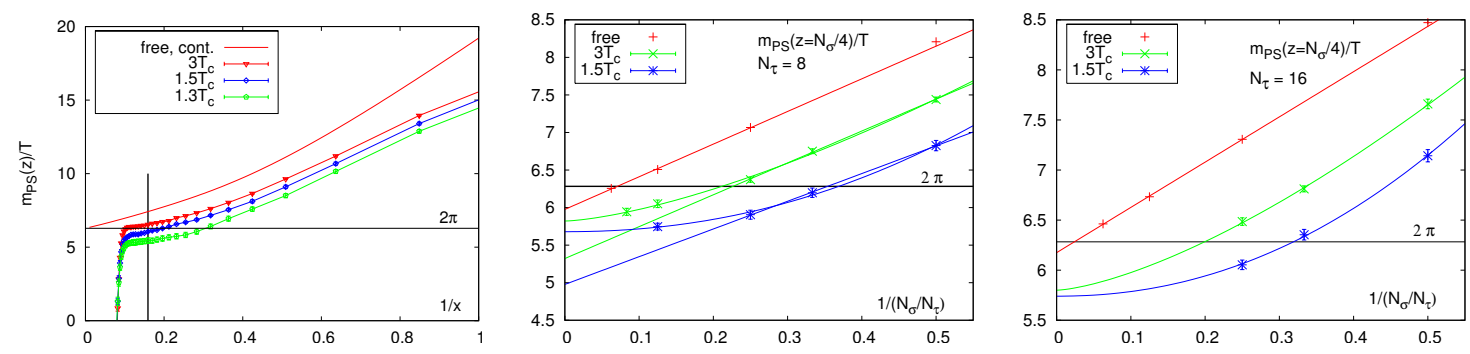

Figure 1: Effective screening masses compared to the free continuum one (left) and as function of aspect ratio (lattice volume) at $N_{\tau}=8,16$

We expect that $p$ takes values between 3 as in the confined phase, e.g. [10], and 1 as in the infinite temperature limit. The fits indeed return results which decrease towards 1 with rising temperature, Table 2.

The remaining lattice spacing dependence is expected to be quadratic in $a$ or, equivalently in $1 / N_{\tau}$

$$
\frac{m(a)}{T}=\frac{m_{\mathrm{scr}}}{T}-\lambda\left(\frac{1}{N_{\tau}}\right)^{2}+\ldots
$$

in the free as well as in the interacting case. As Fig. 2 (left) shows the coefficient $\lambda$ is rather small in the latter. The final numbers are given in Table 2 and in Fig. 2.

\begin{tabular}{c|c|c}
\multirow{2}{*}{$\mathrm{T}$} & \multicolumn{2}{|c}{$p$} \\
\cline { 2 - 3 } & $\mathrm{PS}$ & $\mathrm{V}$ \\
\hline $1.5 T_{c}$ & $2.12(36)$ & $2.24(46)$ \\
$3 T_{c}$ & $1.46(15)$ & $1.54(21)$
\end{tabular}

\begin{tabular}{c|c|c}
\multirow{2}{*}{$\mathrm{T}$} & \multicolumn{2}{|c}{$m_{\mathrm{scr}} / T$} \\
\cline { 2 - 3 } & $\mathrm{PS}$ & $\mathrm{V}$ \\
\hline $1.5 T_{c}$ & $5.77(1)$ & $6.21(1)$ \\
$3 T_{c}$ & $5.81(4)$ & $6.06(4)$
\end{tabular}

Table 2: Results from the data fit to Eq. (3.5) and (3.6)

Due to exceptional configurations it is difficult to analyse the data below $1.3 T_{c}$. As can be seen, the pion screening mass is about $9 \%$ smaller than in the free case while the vector meson comes out close to but also below $2 \pi$. This is to be contrasted with the analytical calculation based
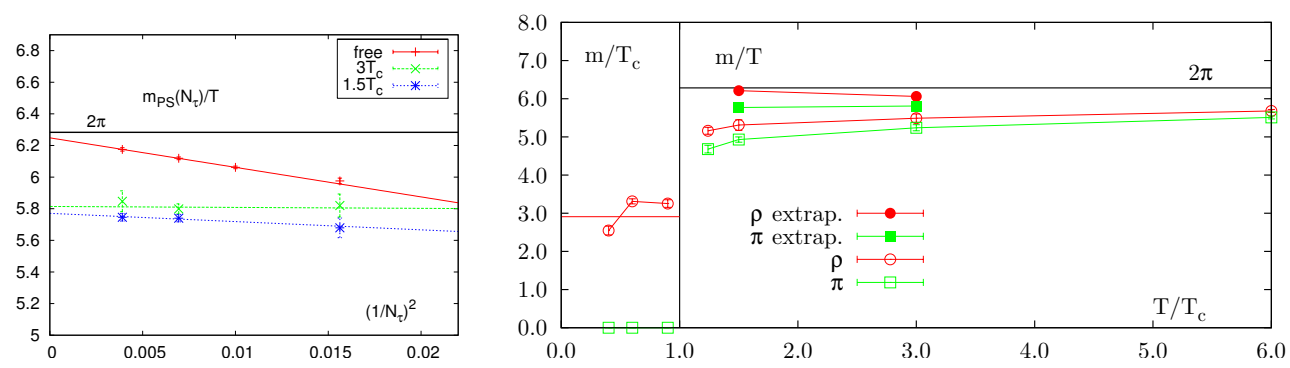

Figure 2: Finite lattice spacing extrapolation (left) and extrapolated result compared to old results, which have been obtained via rescaling with $2 \pi / m_{\text {free }}^{\text {scr }}(L, a)$.

on dimensional reduction and treating $\omega_{0}^{\text {quark }}$ as a heavy quark mass [11], which predicts that the screening masses approach the free result from above. 


\section{Spectral functions for the Wilson vs. the hypercube action}

One important step towards the understanding of in-medium properties of mesons in finite temperature QCD is the study of their spectral functions (SPFs). They are necessary to establish connections with experimental results e.g. the dilepton or photon production rates and their mass spectrum. The computation of the SPFs on the lattice from the given correlation functions is an ill posed problem. However, one may attempt to use the well established MEM [12], which has been first utilized in [13], to tackle the problem. In this method, information about the large $\omega$ part of the SPF, which is not well determined by the data, is supplied as a prior default in the form of a Shannon-Jaynes entropy term. The crucial point is to find a proper default model which serves as an input for MEM. To this end we consider the free lattice SPFs derived in [9, 14, 15] for our fermion actions. In both cases MEM can nicely reproduce them with the input provided by the free correlation functions, see Fig. 3 (left). In the free case the lattice artifacts for the hypercube fermions are pushed to much higher frequencies than for the Wilson fermions. The same behavior is seen also for the interacting case and thus allows for a more reliable study of interesting states in the physical interesting low $\omega$ region, see Fig. 3 (middle). The reconstructed SPFs at $1.5 T_{c}$ and $3 T_{c}$ are significantly different from the free case. The positions of the first and the second bumps for both fermion discretizations are in agreement. The second bump in the case of the hypercube action corroborates, that it may not be a lattice artifact, as one may have thought from studies with Wilson fermions. To solidify the SPF results from the hypercube action and to turn them into predictions for the dilepton spectrum one needs renormalization constants, whose determination is work in progress.
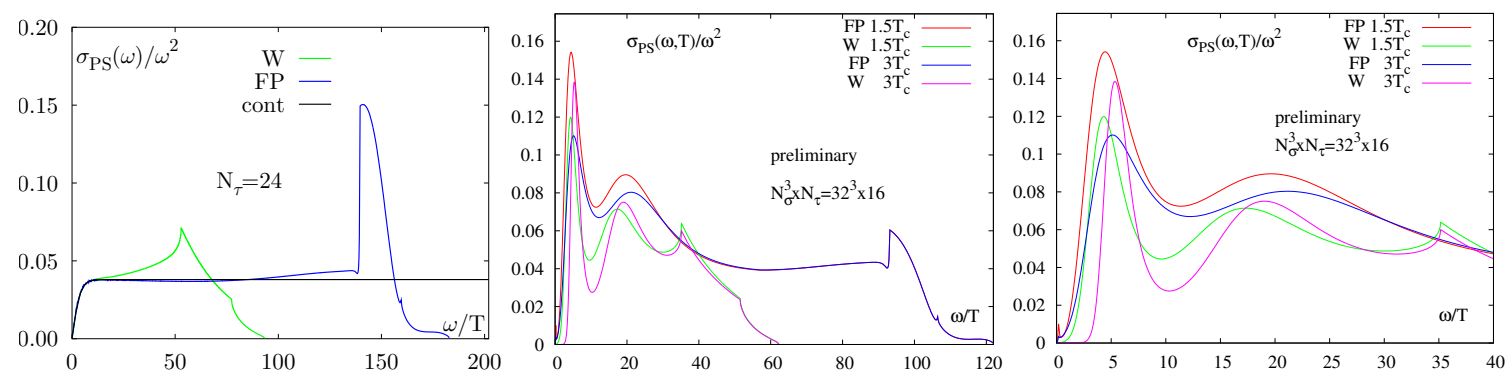

Figure 3: SPFs for the pseudo-scalar channel with Wilson (W) and hypercube (FP) fermions at $T=\infty$ (left) and at $1.5 T_{c}, 3 T_{c}$

\section{Summary}

We have presented an analysis of infinite volume and continuum extrapolations of the effective screening masses for different mesons with the improved Wilson action above $T_{c}$. The resulting values come closer to the free values. SPFs computed with hypercube fermions seem to allow the identification of the excited states with higher confidence than Wilson fermions. To fully confirm this behavior we further need to compute the renormalization constants, improve our statistics and increase the volume. 


\section{Acknowledgments}

The computations have been performed on the IBM-JUMP computer at NIC Jülich. This work was supported by the DFG under grant GRK 881.

\section{References}

[1] R. G. Edwards, U. M. Heller, and T. R. Klassen, Accurate scale determinations for the wilson gauge action, Nucl. Phys. B517 (1998) 377-392, [hep-lat/9711003].

[2] M. Lüscher, S. Sint, R. Sommer, P. Weisz, and U. Wolff, Non-perturbative o(a) improvement of lattice qcd, Nucl. Phys. B491 (1997) 323-343, [hep-lat/9609035].

[3] B. Sheikholeslami and R. Wohlert, Improved continuum limit lattice action for qcd with wilson fermions, Nucl. Phys. B259 (1985) 572.

[4] W. Bietenholz, R. Brower, S. Chandrasekharan, and U. J. Wiese, Progress on perfect lattice actions for qcd, Nucl. Phys. Proc. Suppl. 53 (1997) 921-934, [hep-lat/9608068].

[5] K. G. Wilson, The renormalization group: Critical phenomena and the kondo problem, Rev. Mod. Phys. 47 (1975) 773.

[6] P. Hasenfratz and F. Niedermayer, Perfect lattice action for asymptotically free theories, Nucl. Phys. B414 (1994) 785-814, [hep-lat/9308004].

[7] W. Bietenholz and U. J. Wiese, Perfect lattice actions for quarks and gluons, Nucl. Phys. B464 (1996) 319-352, [hep-lat/9510026].

[8] W. Florkowski and B. L. Friman, Spatial dependence of meson correlation functions at high temperature, Z. Phys. A347 (1994) 271-276.

[9] F. Karsch, E. Laermann, P. Petreczky, and S. Stickan, Infinite temperature limit of meson spectral functions calculated on the lattice, Phys. Rev. D68 (2003) 014504, [hep-lat/ 0303017$].$

[10] M. Fukugita, H. Mino, M. Okawa, G. Parisi, and A. Ukawa, Finite size effect for hadron masses in lattice qcd, Phys. Lett. B294 (1992) 380-384.

[11] M. Laine and M. Vepsalainen, Mesonic correlation lengths in high-temperature qcd, JHEP 02 (2004) 004, [hep-ph/0311268].

[12] M. Asakawa, T. Hatsuda, and Y. Nakahara, Maximum entropy analysis of the spectral functions in lattice qcd, Prog. Part. Nucl. Phys. 46 (2001) 459-508, [hep-lat/ 0011040 ].

[13] Y. Nakahara, M. Asakawa, and T. Hatsuda, Hadronic spectral functions in lattice qcd, Phys. Rev. D60 (1999) 091503, [hep-lat/9905034].

[14] G. Aarts and J. M. Martinez Resco, Continuum and lattice meson spectral functions at nonzero momentum and high temperature, Nucl. Phys. B726 (2005) 93-108, [hep-lat/ 0507004 ].

[15] G. Aarts, On meson spectral functions at high temperature and nonzero momentum, PoS(LAT2005)182 (2005) [hep-lat/0509062]. 